\title{
Entre el cuaderno de comunicados y el smartphone: Los desafíos para la gestión en centros educativos uruguayos en el contexto de comunicación digital
}

\author{
Between the communication notebook and the smartphone. \\ The challenges for the management in Uruguayan \\ educational centers in the context of digital communication
}

https://doi.org/10.22235/d.vi32.2118

\author{
Patricia Silva \\ Universidad Católica del Uruguay, Montevideo, Uruguay \\ ORCID: 0000-0001-9267-3509 \\ Matías Dodel Schubert \\ Universidad Católica del Uruguay, Montevideo, Uruguay \\ ORCID: 0000-0002-1724-9609 \\ Ana Inés Pepe \\ Universidad Católica del Uruguay, Montevideo, Uruguay \\ ORCID: 0000-0003-1704-4088
}

\begin{abstract}
RESUMEN
Este trabajo explora cómo se gestiona la comunicación en los centros educativos con uno de sus stakeholders prioritarios: las familias. Los centros se enfrentan a un entorno que combina un uso masivo de comunicación digital por parte de las familias, junto a una importante varianza en los contextos socioeconómicos, políticas organizacionales y liderazgos. Se presenta un estudio de caso de una red de doce colegios privados en Montevideo (Uruguay), en los que se realizaron entrevistas semiestructuradas a directores y maestros. Los hallazgos señalan que, a pesar de la masificación de dispositivos digitales, directores y docentes continúan expresando preferencias por canales tradicionales para la mayoría de los emergentes comunicacionales. Aún sin un rechazo explícito a las tecnologías digitales, aparecen diferentes barreras que impiden su implementación. Se brindan recomendaciones para repensar la comunicación a partir de las estrategias organizacionales.
\end{abstract}

Palabras clave: comunicación digital; competencias comunicacionales; escuela; liderazgo; desigualdad social.

\begin{abstract}
This article assesses how schools manage their communication with students' families. Schools face a challenge that combines the massive use of digital communication by families, considerable socioeconomic variance in their educational contexts, and a diversity of organizational policies and leadership styles. Based on a case study of a network of twelve private schools in Montevideo, we conducted semi-structured interviews with principals and teachers. Our findings indicate that, despite the massification of digital devices, principals and teachers continue to express preferences for traditional non-digital channels of communication, for the majority of school-related topics. Even without an explicit rejection of digital technologies, respondents manifest diverse barriers that prevent their implementation. Recommendations are provided for rethinking communication based on organizational strategies.
\end{abstract}

Keywords: digital communication; communication skills; school; leadership; social inequality.

Cómo citar: Silva, P., Dodel Schubert, M., y Pepe, A. I. (2020). Entre el cuaderno de comunicados y el smartphone: Los desafios para la gestión en centros educativos uruguayos en el contexto de comunicación digital. Dixit, 32, 61-75. https://doi.org/10.22235/d.vi32.2118 
Introducción y estado de la cuestión

El presente trabajo explora y describe cómo una red de centros educativos privados de Montevideo (Uruguay) gestionan su comunicación con uno de sus stakeholders (públicos de interés) prioritarios: las familias de los estudiantes. Es de particular relevancia para el estudio de esta temática el contexto actual de estos centros: un uso masivo de comunicación digital por parte de las familias, junto a importante varianza en los contextos socioeconómicos de los centros y las familias, estilos organizacionales y de liderazgo en cada institución. El contexto de emergencia sanitaria generado por la pandemia de covid-19 ha puesto en evidencia la importancia y la urgencia de desarrollar competencias en el uso de tecnología de comunicación digital para sostener la interacción, aún más en períodos de aislamiento.

\section{Comunicación con stakeholders estratégicos en los centros educativos}

La gestión de la comunicación de los centros educativos con sus stakeholders es, como en organización, de relevancia estratégica (Forero, 2011; Mayo y García, 2012). Una institución educativa que no planifica con seriedad, respondiendo a las demandas o expectativas de sus stakeholders, en los dinámicos tiempos actuales, se expone ante el riesgo de perder o distanciarse de estos actores clave en momentos críticos (Lingam, Lingam, y Raghuwaiya, 2014). A modo de ejemplo, en el contexto de la pandemia de covid-19, Reimers y Schleicher (2020) sostienen que el desarrollo de un plan de comunicación para el relacionamiento con los stakeholders resulta central para dar una respuesta educativa apropiada frente al aislamiento, de modo que los mensajes prioritarios para sostener la estrategia educativa puedan llegar a las familias.

A pesar de la relevancia de las familias, son escasas las investigaciones acerca de la comunicación de las organizaciones educativas con estas. Los estu- dios sobre este punto se enfocan principalmente en la comunicación para promover la participación de las familias de modo de impactar en el proceso de enseñanza-aprendizaje (Bordalba, 2016; Epstein, 1995; LaRocque, Kleiman, y Darling, 2011). La literatura especializada señala a la comunicación entre escuela y familia como uno de los aspectos clave para favorecer el alcance de los logros educativos significativos (Epstein, 1985; El Nokali, Bachman y Votruba-Drzal, 2010; Bochaca, 2015). Consecuentemente, una de las demandas más sentidas por las familias para incrementar su participación es la de mantenerlas informadas (Guardia Romero y Santana Armas, 2010).

Epstein (2013) sostiene que es clave la construcción de una alianza con las familias, y presenta como uno de los desafíos el generar comunicaciones "bidireccionales" claras entre el hogar y la escuela. Sin embargo, Bordalba (2016) plantea que las herramientas bidireccionales se siguen utilizando poco, ya que en la mayoría de los casos su adopción depende de la decisión individual del maestro.

Asimismo, los estudios organizacionales dan cuenta de que el estilo de relaciones, su efectividad y la comunicación con los stakeholders en las escuelas tienen que ver con los estilos de liderazgo (Bush, 2007, Odhiambo y Hii, 2012). En los centros educativos la comunicación es el medio para crear un lenguaje y una visión compartida también con las familias, función específica de un líder (Beauchamp y Parsons, 2014). No obstante, los liderazgos se configuran en una cultura organizacional conservadora, en la que es muy difícil introducir cambios, y se alimentan de una estructura piramidal de poder y privilegios (Hodas, 1996; Gore, 2004; Engen, 2019). Estos factores característicos son los que, según Hodas (1996), contribuyen al rechazo hacia la incorporación de las tecnologías para la comunicación digital en las escuelas. 
TIC, desigualdad digital y comunicación mediada por computadora en los centros educativos

Las tecnologías de la información y la comunicación (TIC) poseen un potencial nunca visto hasta el momento para la democratización del acceso, consumo y difusión e intercambio de información a niveles globales. No obstante, estas potencialidades no siempre se concretan en realidades y se generan importantes desigualdades entre quienes las pueden aprovechar adecuadamente y quienes no (Peña-López, 2009). La dependencia creciente de las TIC en la actualidad refuerza inequidades ya existentes, como por ejemplo de género, nivel socioeconómico o etnia (Robinson et al., 2015).

En el ámbito de la comunicación, las TIC han generado importantes cambios en la forma de relacionarse entre las personas. Según Walther (1996), la comunicación mediada por computadora (CMC) es sostén de todas las relaciones interpersonales y se la puede observar en todos los contextos relacionales. Para el autor, esta puede provocar las mismas percepciones que en la comunicación cara a cara, como el feedback o la cercanía psicológica, si los contextos son los adecuados (Korzenny citado por Walther, 1996). Sin embargo, algunos atributos de la CMC generan cambios en la forma de relacionarse, como la posibilidad de la comunicación asincrónica, la controlabilidad, la registrabilidad y las diferentes formas de direccionalidad de la comunicación (Walther, 2011).

El contexto de uso masivo de comunicación digital es una oportunidad para los centros educativos. Tanto para Hohlfeld, Ritzhaupt y Barron (2010) como para Cox y McLeod (2014) las escuelas pueden aumentar la comunicación con las familias y toda la comunidad a través de estos canales. Goodall (2016) reconoce que estas tecnologías bien gestionadas, sobre la base del respeto recíproco entre docentes y padres, facilitan la comunicación bidireccional. También afirma que deberían ser los directivos los que lideran la capaci- tación de los docentes para su uso. Las TIC son una herramienta no opcional para un liderazgo efectivo en el siglo XXI (Cox y McLeod, 2014). Olmstead (2013) sostiene que el modo de mejorar la participación de los padres y el rendimiento de los alumnos, en un contexto de jornadas laborales muy extensas, es la utilización de los medios electrónicos de comunicación. Sin embargo, varios autores acuerdan que este potencial de las TIC para la comunicación no se aprovecha en su totalidad.

Por otro lado, resulta evidente que se han incrementado los canales de comunicación entre docentes y padres (Wasserman y Zwebner, 2017). Los teléfonos inteligentes conectados a Internet permiten comunicarse desde cualquier lugar y en cualquier momento, derrumbando algunas de las barreras de disponibilidad, tiempo y espacio que diferenciaban el tiempo escolar de la vida del hogar (Wellman et al., 2003; Cox y McLeod, 2014; Wellman y Hogan, 2004). Noblía (2018) sostiene que son los dispositivos móviles los que actualmente concentran la interacción interpersonal en las organizaciones y conforman redes sociales dinámicas, como sucede con WhatsApp. De acuerdo con Ersöz (2019), por ejemplo, resulta difícil encontrar colegios sin grupos de WhatsApp de padres muy activos. La práctica y la literatura indican que los docentes suelen también formar parte de los grupos de WhatsApp de la clase (Bouhnik, Deshen, y Gan 2014; Hernández, Martínez y Vicente, 2017) y que hay escasa regulación de los comportamientos de estos grupos, principalmente entre padres.

Limitantes como la ausencia de regulación generan conflictos y dificultades de comunicación con los centros (Hernández et al., 2017). Asimismo, existen una serie de barreras de peso para alcanzar un adecuado aprovechamiento de las TIC para la comunicación. Según Aragonés (2001), se requiere un mayor desarrollo de la competencia comunicativa de los docentes. 
Para Engen (2019) no se han desarrollado dichas competencias debido a la lentitud frente a los cambios y adaptación a la tecnología en las escuelas, así como a las frecuentes conductas conservadoras de los docentes en relación con las TIC.

Pieri (2018) argumenta que los propios docentes sostienen no haber sido formados en la utilización de las TIC en general, y menos en su uso para la comunicación con las familias. Fraillon, Ainley, Schulz, Friedman y Duckworth (2019) - a partir del estudio comparado internacional ICILS sobre competencias digitales e informacionales en estudiantes de octavo ciclo (tercer año de liceo en el sistema educativo uruguayo- sostienen que los maestros jóvenes tienden a tener más confianza y habilidades en el uso de las TIC, aspectos que determinan directamente el cómo las aplican en clase y en la relación con sus alumnos.

Por último, es importante considerar -sobre todo a raíz de la pandemia de covid-19- la relevancia de las herramientas digitales para el aseguramiento de los procesos cotidianos de gestión de los centros educativos (Banco Interamericano de Desarrollo, 2020). Tanto en su mejora de la gestión interna de la organización como en la facilitación de la comunicación con las familias, al descongestionar las cargas administrativas de los docentes, las TIC también colaboran en el incremento del tiempo directo destinado a enseñar (Banco Interamericano de Desarrollo, 2019).

\section{Preguntas de investigación e hipótesis}

Este estudio tuvo como finalidad describir cómo los centros educativos gestionan su comunicación con uno de sus stakeholders prioritarios -las familias- en un contexto de uso masivo de comunicación digital, disparidades socioeconómicas y organizacionales. Las preguntas de investigación se centraron en cómo conviven los diferentes tipos de canales de comunicación en las relaciones centro-familia: ¿Existen preferencias por canales a partir de diferentes tipos de emergentes comunicacionales? ¿Qué barreras existen para la adopción de medios digitales? ¿Qué factores organizacionales pueden condicionar la utilización de los diferentes canales? ¿Cómo afectan las desigualdades socioeconómicas de contexto a las prácticas de comunicación y preferencia por lo digital?

Consecuentemente, se plantean las siguientes cinco hipótesis:

- H1: Dada la falta de sensibilización en los centros sobre el rol de la comunicación organizacional, no se encontrarán protocolos para la comunicación digital con las familias.

- H2: Dada la ausencia de protocolos, el liderazgo es uno de los factores preponderantes para determinar las prácticas comunicacionales.

- H3: Dado que la escuela tiende a ser conservadora en sus prácticas, se encontrará preferencia por los canales tradicionales.

- H4: El bajo nivel de desarrollo de las competencias digitales y comunicacionales de los maestros y directores será una de las barreras a la adopción de canales digitales.

- H5: En contextos socioeconómicos más deprimidos se espera encontrar barreras en el uso de las TIC con los centros.

\section{Método}

Para responder a las preguntas de investigación se realizó un estudio de caso de una red de centros educativos en Montevideo (Uruguay), organización de segundo nivel encargada de coordinar una gestión pedagógica y administrativa común. La red seleccionada como caso de estudio, compuesta por 12 centros privados en la capital y 12 en el interior, cuenta con más de 4000 alumnos que 
van desde preescolar (2 años) hasta bachillerato (17 años). El 74\% de los alumnos reside en Montevideo. Para proteger la confidencialidad de los entrevistados se eliminó todo dato que permitiera identificar a los centros y participantes.

El estudio es cualitativo, exploratorio-descriptivo, y la recolección de información se hizo a partir de 36 entrevistas a directores y maestros, con una pauta semi-estructurada. Se censó a todos los centros y directores de Montevideo y se determinó una muestra teórica dentro de estos, seleccionando una submuestra de maestros. Se eligieron, por cada centro, maestros que tuvieran a cargo los grupos del primer y último año de primaria, niveles que al encontrarse en las fronteras del ciclo implican una mayor interacción entre el centro y las familias. Se realizaron 12 entrevistas a los directores de los 12 centros de la red y 24 entrevistas a los docentes, uno de primer y otro de último año de primaria, en cada centro.

La selección de la red como estudio de caso se debe a tres motivos principales. Por un lado, la facilidad de acceso a los 12 centros a través de un único punto de contacto con la organización central. En segundo lugar, la red es relevante para el objeto de estudio debido a que los centros que la componen se encuentran en un momento singular de implementación de una aplicación digital, diseñada específicamente para facilitar la comunicación con las familias a través de la telefonía móvil. Por último, la distribución de los centros de la red en contextos socioeconómicos muy variados permite, aún dentro de las limitaciones de esta selección intencional del caso, contar con una suerte de muestra teórica en lo que refiere a la diversidad socioeconómica de los contextos de los centros educativos privados en Montevideo.

En este sentido, la Tabla 1 presenta la distribución de los centros por barrios, en función del porcentaje de personas con alguna necesidad básica insatisfecha (NBI).
Tabla 1

Cuadro de distribución de los centros en Montevideo, con porcentaje de personas con al menos una NBI

\begin{tabular}{ccc}
\hline $\begin{array}{c}\text { N. }{ }^{0} \text { de } \\
\text { centros }\end{array}$ & $\begin{array}{c}\text { \% de personas con } \\
\text { al menos una NBI }\end{array}$ & $\begin{array}{c}\text { Grupo } \\
\text { de NBI }\end{array}$ \\
\hline 2 & 60,1 & Alto \\
\hline 2 & $47,3-52,8$ & Medio-Alto \\
\hline 3 & $31,9-33,5$ & Medio \\
\hline 3 & $21,10-27,5$ & Medio-Bajo \\
\hline 2 & $7,10-16,3$ & Bajo \\
\hline
\end{tabular}

Fuente: Elaboración propia en base a Calvo (2013), según datos del último censo poblacional de Uruguay (2011)

A partir de la literatura revisada se construyó una pauta de entrevista para directores y docentes que indagaba: cuáles eran las situaciones o emergentes que generaban en los centros la necesidad de comunicarse con las familias; cuáles eran los canales elegidos para hacer efectiva esa comunicación; qué tan planificadas y reguladas eran esas interacciones y a cargo de quién estaban; cómo era su nivel de desarrollo de competencias digitales y cómo había sido la experiencia de tener una aplicación para teléfonos celulares desarrollada para comunicarse con las familias en esta red de centros. Las entrevistas se analizaron a partir de una matriz en la que se clasificaron las respuestas de los docentes por un lado y directores por otro; de docentes menores de 40 y mayores de 40; y de entrevistados de acuerdo a los diferentes contextos socioeconómicos, identificando similitudes y diferencias en sus respuestas. 


\section{Resultados}

El análisis de las entrevistas permitió validar los postulados de la literatura en relación con los diferentes factores que impactan en la gestión de la comunicación de los centros con las familias: la ausencia de planificación en este aspecto y el estilo de liderazgo de cada director. Además, se identificaron qué factores impactan en la elección de canales para la comunicación con las familias: preferencia por canales unidireccionales tradicionales, a pesar de las ventajas percibidas en los medios digitales bidireccionales, desigualdades socioeconómicas y debilidades en las competencias digitales de directores y maestros.

\section{Factores que afectan la gestión}

\section{de la comunicación con las familias}

Entre los factores que impactan en la comunicación con las familias se encontró la falta de un protocolo para la comunicación con estas, lo cual permitió evidenciar los variados matices en las orientaciones dadas a los docentes para su gestión, según los estilos de liderazgo de los directores de los diferentes centros.

\section{Ausencia de protocolos de comunicación}

Se observó la ausencia de protocolos escritos para la comunicación con las familias, tanto a nivel centro como red. No obstante, se constató que los directores dan pautas u orientaciones verbales sobre la comunicación con las familias, principalmente al inicio del año, que fundamentalmente tienen como objetivo prevenir conflictos:

La directora nos dijo qué cosas se podían comunicar a través del cuaderno, qué cosas a través de la aplicación, qué es mejor hablar en persona. Que cada vez que había algún conflicto con algún niño siempre está bueno contárselo a los padres. Porque el niño va y cuenta una versión. Contar la versión general a los dos padres (maestra de $1^{\circ}$, NBI bajo, 23 años).
Sí, se trabaja mucho qué hacer frente a cada situación. Tratar de no estar solas en las entrevistas donde hay algún conflicto. Tomar nota de lo que se habló y hacerlo firmar (maestra de 6º, NBI bajo, 59 años).

La ausencia de perspectiva sobre la comunicación organizacional, junto las orientaciones muy generales de los directores, se observaron en todos los centros. En el discurso de los directores siempre está presente la intención de proteger a los docentes y a la institución de posibles malos entendidos o riesgos que puedan agravarse por una inapropiada gestión de los canales que se utilizan:

A veces en la reuniones se trata el tema, pero no sobre "la comunicación". Que tengamos cuidado [...] que hay que consultar. No responder con agresividad, escribir lo menos posible, invitar a una entrevista o llamar por teléfono (maestra de $6^{\circ}$, NBI medio alto, 45 años).

Sin embargo, estas recomendaciones que tienen el fin de proteger a los docentes -o la función docente- a veces son percibidas por los maestros como generadoras de una distancia innecesaria con las familias, o como indicadoras de una resistencia a los cambios y el arraigo a las formas más tradicionales de comunicación:

Me gustaría acercar un poco más a las familias. Siento que siempre se marca una cierta distancia. Creo que es necesario poder trabajar a la par con el hogar de cada niño. A mí me gustaría acercarlos más (maestro de $1^{\circ}$, NBI medio alto, 31 años).

Quizás la directora la ve (la aplicación) como muchos cambios y no quiere agregar algo más (maestra de $1^{\circ}$, NBI medio alto, 27 años). 
En síntesis, si bien la comunicación y alianza con las familias se considera como un aspecto crítico para el cometido de los centros (Epstein, 2013), lo manifestado por los entrevistados evidencia la falta de planificación de una estrategia de comunicación institucional (Forero, 2011; Mayo y García, 2012).

\section{Estilos de liderazgo}

A partir de una ausencia de políticas o protocolos establecidos para toda la red, el tipo de liderazgo ejercido por la dirección influye directamente en las prácticas de comunicación.

La introducción de la aplicación en la red de colegios permite visibilizar claramente el efecto de los estilos diferenciales de liderazgo. Si bien es una herramienta poderosa para la comunicación entre los docentes y las familias, no todos los directores la consideran una prioridad. Algunos directores declaran que ya la utilizan para la comunicación institucional unidireccional, pero aún no utilizan la función que permite la comunicación de los maestros con las familias, al no ser prioritaria desde su perspectiva:

La aplicación no [no la gestionan los maestros] porque el director general no quiso [si bien la directora de primaria sí] porque él considera que se pueden equivocar. Solo los directores la manejan (directora, NBI medio, 49 años).

La app la manejo yo [...], es el director el responsable de esa información que sale [...]. Y hoy por hoy sabemos que tenemos docentes en los que podemos confiar en su expresión, en su ortografía o en las cosas que responden, ciegamente, y hay otros que no (directora, NBI bajo, 38 años).

Asimismo, el estilo de dirección también impacta en la utilización de los medios tradicionales, como es el control de los comunicados a las familias. Por ejemplo, algunos directores supervisan la totalidad de estos intercambios:
Tratamos de que los comunicados en forma general pasen por dirección. Cuando hay una carta de un papá que sorprende un poco, o tiene algunas puntas áridas, el comunicado pasa por dirección y con el docente tratamos de elaborar una respuesta. Si es un comunicado donde hay que dar alguna respuesta, que pase por dirección (directora, NBI medio bajo, 52 años).

Mantener los límites claros. Hay un apoyo, un aconsejarnos. Antes de mandar un comunicado muéstrenmelo. Yo trabajo con la directora desde hace dos o tres años, y sabe cómo trabajo, cómo me comunico con las familias o con las instituciones, por lo que en ese sentido no [se] necesita de algún protocolo o una cosa así (maestro de $6^{\circ}$, NBI alto, 35 años).

Los liderazgos con tendencia a la centralización controlan el uso de los canales de comunicación digital con la finalidad de evitar riesgos, ya que consideran que sus características de inmediatez y alto impacto pueden tener consecuencias negativas:

Hubo una maestra que tenía grupo de WhatsApp con los padres, pero cuando yo ingresé le hice presente que nosotros no teníamos esa forma de comunicación oficial, que no me parecía adecuada y salió del grupo. Por las situaciones que había aquí, no era recomendable porque se generaban conflictos, y algunos docentes fueron llamados a su teléfono particular en horarios de otro trabajo o en momentos de familia (directora, NBI medio alto, 44 años).

Cuando los maestros tienen grupos de WhatsApp con los padres yo tiemblo [...] porque uno contesta sobre la inmediatez y queda registrado (directora, NBI medio, 47 años). 
Cuando los estilos de dirección son menos centralizadores, se deja libertad de acción para el uso de las redes sociales para la comunicación con las familias. El riesgo para los maestros es no contar incondicionalmente con el respaldo de la dirección:

Yo les dije que quien quisiera tenerlos [por grupo de WhatsApp con padres] que los tenga. Yo no puedo mandar sobre la libertad individual de cada uno, pero que yo no respaldo lo que suceda (directora, NBI medio, 47 años).

Por último, la no utilización de herramientas digitales se percibe también como una resistencia del sector:

Me encanta, me gusta mucho manejarme en el tema tecnológico, a nivel personal tengo muchas aplicaciones en el celular. Me es difícil [...] en mi práctica docente aplicar el tema de la tecnología, por un montón de motivos. No está instalado en el ámbito [educativo] privado (maestra de $6^{\circ}$, NBI medio, 22 años).

En suma, lo expuesto en las entrevistas se condice con lo que la literatura señala sobre la prevalencia de una cultura organizacional conservadora y piramidal en la estructura de poder de los centros educativos (Hodas, 1996; Gore, 2004; Engen, 2019). Estas estructuras, a su vez, son moderadas a través del estilo de liderazgo de cada director. Aún con cierta variación, los liderazgos se corresponden con esta estructura piramidal, lo que se refleja en una clara inclinación hacia la centralización y control directo de las comunicaciones con las familias.

Dado este contexto, existen más resistencias a innovar en la comunicación con las familias, especialmente en aspectos vinculados a la tecnología digital que, tal como señala Hodas (1996), son percibidas como un riesgo al modificar las prácticas tradicionales de interacción. A modo de ejemplo, una herramienta de comunicación digital eficaz y potente como lo es la aplicación institucional no fue ampliamente apropiada en la red por temor a la introducción de cambios (Hodas, 1996; Gore, 2004; Engen, 2019). Si bien la decisión política de habilitar su uso cae en manos de las direcciones (Bordalba, 2016), las barreras para una implementación exitosa no son exclusivas de estas: los docentes tampoco son proclives a incorporar las TIC en las escuelas (Hodas 1996).

Factores que afectan la elección de canales de comunicación con las familias

Los hallazgos demuestran que se perciben las ventajas de la comunicación digital, pero algunos elementos siguen incidiendo para dar continuidad a la preferencia por los canales tradicionales unidireccionales, la cual es reforzada por los condicionantes de las desigualdades socioeconómicas y las debilidades en competencias digitales.

Preferencia por canales tradicionales unidireccionales A pesar de percibir ventajas en los canales digitales, los tradicionales como el cuaderno de comunicados y la entrevista personal aparecen como los canales preferidos para el tratamiento de situaciones particulares, como los problemas de rendimiento o de conducta:

Siempre usamos, desde tiempos remotos, el cuaderno de comunicados. Ni me acuerdo desde cuando... ¿Indicaciones? Hay pautas sí, lo normal que se escribe porque es desde siempre (maestra de $6^{\circ}$, NBI medio bajo, 54 años).

La preferencia por un medio tradicional, como es el cuaderno de comunicados, se funda en la seguridad que se percibe en lo que queda registrado en soporte papel, lo que desconoce una de las características propias de la CMC: toda interacción queda registrada. Se aprecia, además, como más práctico para recordar los acuerdos a las familias, cuando sea necesario: 
Los mismos padres pidieron que no se rompiera el vínculo a través del cuaderno de comunicados. [...]. Y también confiar. El papel les da seguridad. Obviamente todo lo que tenga que venir firmado... autorizaciones... el papel está (directora, NBI bajo, 38 años).

Las entrevistas personales se ubican en el segundo lugar de preferencia para la comunicación con las familias. Estas son con el maestro a cargo del grupo, con o sin la presencia del director.

Insistimos en cómo tener las entrevistas con los padres. Que pidan ayuda (directora, NBI medio alto, 40 años).

Este año empezamos a utilizar el modelo de entrevistas, que siempre dejemos registrado todo lo conversado con la firma de los padres o el entrevistado (maestro de $1^{\circ}$, NBI medio alto, 27 años).

La alternativa a los medios tradicionales como el cuaderno o la entrevista, para asegurar la llegada de un comunicado, en todo caso suele ser el teléfono institucional de línea:

Tenemos 600 alumnos y estamos utilizando la app, pero para algo que querés asegurarte que le llegó, teléfono (directora, NBI medio alto, 40 años).

Lejos de comprender la utilidad de la controlabilidad, asincronicidad y la registrabilidad propia de los medios digitales (Walther, 2011), existe una creencia entre docentes y directores sobre su falibilidad y riesgos (Hodas, 1996). Estos factores inclinan las preferencias por "la seguridad del papel", así como la cercanía de las entrevistas personales. Ambos canales tienen una serie importante de limitantes, mencionadas también por los entrevistados, en términos del tiempo con el que cuentan los padres o la imposibilidad de asegurarse la lectura de los cuadernos de comunicados en tiempo y forma. Si bien no fue considerado por los entrevistados dado el momento del estudio, es necesario señalar que estos canales son extremadamente endebles -por no decir inviables- en contextos de emergencias naturales o sanitarias.

\section{Ventajas percibidas en la comunicación digital}

Si bien los canales digitales no son los predilectos, se advierte que para los directores y maestros más jóvenes pueden ser la solución a los problemas que presentan los canales tradicionales. Es constatable la facilidad con que se pueden gestionar las herramientas digitales y cuánto más prácticas son en lugar del cuaderno de comunicados:

En los cuadernos de comunicados las cosas salen sobre la hora y a veces casi no tenés tiempo de pegar el comunicado cinco minutos antes (de la salida), cuando ya guardaron el cuaderno. [...] Quizás es por un tema de seguridad de la directora, que está más arraigada al papel. [...] Yo estoy abierta, a mí me resulta muy fácil [la comunicación digital] (maestra de $1^{\circ}$, NBI medio alto, 27 años).

Algunos problemas provocados por los medios de comunicación tradicionales ponen de relieve la ventaja que significaría la utilización de medios digitales. Por ejemplo, en el caso de un centro con horario extendido -donde el grupo de alumnos tiene más de un docente a cargo entre la mañana y la tarde-, y en los que los docentes no estaban al tanto de los mensajes emitidos en el otro turno, la aplicación les permitió recibir los comunicados en el mismo momento en que se envían a los padres:

El tema del cuaderno de comunicados es de mucha ida y de demanda. Se emite el comunicado, va el comunicado pegado, pero depende de la maestra que está cuando se emite el comunicado, asegurarnos que ese comunicado vaya 
pegado, sea leído y vuelva firmado [...]. Quizás la que envía el comunicado no es la que recibe la respuesta al otro día. [...] La aplicación de la red ayudó a resolverlo. Permitió unificar la llegada de la información [también a los docentes] (directora, NBI medio bajo, 47 años).

Los conflictos que se pueden crear en la comunicación con las familias a través de las redes sociales es, para los docentes más jóvenes, un tema a debatir. Queda claro que no todas las experiencias han sido negativas. En ocasiones las redes habilitaron organización y creación de comunidad con los padres:

Me parece positivo [el tener grupos de WhatsApp de padres]. Me ha tocado en años anteriores, cuando no estaba todo esto como masivo, que los padres no se conocían entre ellos (maestra de $1^{\circ}$, NBI medio, 22 años).

Me gustaría no quedarnos en lo básico [en cuanto a las TIC]. Que pudiéramos aprovecharlo más, con la comunidad, para utilizarla con la familia (maestra de $1^{\circ}$, NBI medio alto, 31 años).

[Por el WhatsApp de padres] Me dijeron: qué suerte que está el grupo, el cuaderno estaba incompleto y me mandaron foto del cuaderno (maestra de $1^{\circ}$; NBI bajo, 23 años).

En línea con la literatura especializada, los docentes y directores son conscientes sobre los potenciales de las TIC para la comunicación (Walther, 2011) y sus consecuencias en la reducción de los tiempos de gestión (Banco Interamericano de Desarrollo, 2019), más allá de los reparos o miedos que les despiertan. Del mismo modo, las entrevistas también validan que los docentes jóvenes son los que se encuentran más cómodos utilizando las herramientas de comunicación digital y demuestran más confianza en sus habilidades para usarlas (Fraillon et al., 2019). De aquí que para ellos el uso de las redes (como los grupos de WhatsApp) no les resulte un riesgo en sí mismo y se encuentren más afines a su utilización. Sin embargo, no todos los directores habilitaban este canal de comunicación.

\section{Desigualdad socioeconómica}

Las desigualdades socioeconómicas también afectan la utilización de los canales de comunicación digital. La mayor proporción de los centros de esta red se encuentran en contextos de alta vulnerabilidad social. Una directora relata que han intentado compartir algunos de los proyectos (como materiales didácticos) con las familias y no ha resultado porque estas no acceden al correo electrónico:

Lo que pasa es que para que las familias tengan un contacto digital con nosotros tienen que formarse. [...]. El año pasado les mandábamos (materiales) a las direcciones de correo para compartir los proyectos de los chiquilines [...], pero resulta que no lo usaban, debido a que no lo saben usar (directora, NBI medio alto, 40 años).

Del mismo modo, la implementación de la aplicación de la red se ve demorada o imposibilitada por las barreras económicas, que hacen que los padres cambien de proveedor de telefonía móvil o pasen períodos de tiempo sin celular de manera frecuente, principalmente porque no logran, por ejemplo, cancelar una deuda con el proveedor del servicio:

No tenemos la app. La app de la red no está instalada. Es un contexto donde el celular se cambia muy seguido [...] porque lo perdieron, se lo robaron, no lo pueden pagar. En la [plataforma educativa] hay que estar actualizando eso todo el tiempo. Por eso tenemos el número del tío, del abuelo, del primo... (directora, NBI alto, 49 años). 
Otra de las barreras, ligada a las desigualdades socioeconómicas, es la falta de capacidad de los celulares de estas familias y docentes, tanto para recibir correos electrónicos como para bajar la aplicación de la red, debido a que utilizan los modelos más económicos del mercado $\mathrm{y}$, por lo tanto, los que tienen menos prestaciones:

Este es un medio socio económico medio bajo y todavía hay gente que no cuenta con un celular con espacio para tener una aplicación más (directora, NBI bajo, 38 años).

Simpatizo con la tecnología mucho. No utilizo demasiadas aplicaciones en el teléfono porque siempre tengo algún equipo de gama media, pero sí todas las básicas de Google las sé utilizar bien (maestro de $6^{\circ}$, NBI alto, 35 años).

Los testimonios de los docentes y directores expuestos aquí no hacen más que validar la noción de que los potenciales de las TIC para el bienestar no se realizan cuando existen fuertes desigualdades socioeconómicas y digitales de por medio (Robinson et al., 2015). Aspectos muy básicos como la diferencia en la calidad de dispositivos móviles, de baja gama entre las familias de menores recursos y también de algunos docentes, segmentan fuertemente los beneficios de la comunicación a través de las TIC entre los centros de diverso contexto socioeconómico. En otras palabras, incrementan aún más las desigualdades preexistentes (Peña-López, 2009).

\section{Debilidades de las competencias digitales}

\section{de directores y maestros}

Se visibilizaron también debilidades no solo en cuanto al acceso, sino a las competencias digitales de directores y docentes. Estas brechas provocan dificultades para el entrenamiento y un uso básico de la tecnología. En un centro donde la única computadora es la de la sala docente, se relató lo siguiente:

\begin{abstract}
Ahora tengo que entrar [registrar] los carné, tengo que entrar a la página [por la plataforma educativa] y no me acuerdo. [Desearía] que se pudiera hacer por celular, que sea más sencillo (maestro de 6º NBI alto, 35 años).
\end{abstract}

Se detecta que la formación tecnológica en la profesión, aún de las nuevas generaciones docentes, no les permite sentirse habilitados y capaces de su utilización:

Me cuesta integrarlo como un recurso de aula. Nos faltó formación de calidad, porque a veces hemos tenido algunos cursos donde estuvieron [toda] una clase para enseñarnos cómo enchufar un proyector... [en el profesorado] (maestro de 6, NBI bajo, 29 años).

Soy joven, pero me cuesta. Considero que no tuve una muy buena formación. En secundaria no tenía informática y en magisterio la educación fue muy escasa relacionada a las nuevas tecnologías. Sé que tengo ese debe (maestra de 6 , NBI bajo, 29 años).

Asimismo, la falta de familiaridad con las redes sociales tampoco favorece su uso. Experiencias negativas a causa de debilidades en el manejo de la seguridad, entre otras, han influido en algunos casos en la resistencia a su utilización:

No soy afín a las redes [...]. En Facebook particularmente tuve una mala experiencia, porque me habían hackeado el perfil, siendo directora [...]. Yo dije: nunca más, porque no me interesa. Pero cuando llego acá me entero de que soy yo quién tengo que manejar eso (directora, NBI medio bajo, 47 años).

Finalmente, tanto directores como docentes señalan que la escasa capacitación recibida en TIC ha sido para 
su aplicación en el aula, pero nunca en función de sus potencialidades comunicacionales:

La tecnología desde lo que es educativo la he incorporado, me he formado a partir de cursos, para poder incorporar lo que son las prácticas dentro del aula. Y después como ciudadana del mundo me he debido incorporar a lo que son las redes, pero como una necesidad educativa, para no estar aislada de lo que pasa en la vida de los chiquilines (directora, NBI bajo, 38 años).

Tal como señalan estudios comparados a nivel internacional, muchos de los entrevistados se perciben con escasas competencias TIC, aspecto que limita su uso dentro y fuera del aula (Fraillon et al., 2019). Si bien se han propuesto cambios en la currícula de formación docente en lo relativo a competencias digitales, estos cambios van a ritmos significativamente más lentos que los tecnológicos. La formación en TIC declarada por los docentes entrevistados, tanto en lo operativo como comunicacional, es escasa o nula, aspecto que no colabora con la adopción de estas tecnologías (Frailon et al., 2019; Pieri, 2018).

\section{Discusión}

Las entrevistas permitieron verificar las cinco hipótesis. Si bien los directores frecuentemente brindan orientaciones generales a los docentes sobre cómo mantener la comunicación con las familias, esta prácticamente se deja librada a la intuición y experiencia previa de cada docente, evidenciando ausencia de planificación y protocolos formales para la comunicación con estas.

Los estilos de liderazgo de los directores inciden directamente en cómo se gestionan los canales de comunicación, en la determinación de cuáles se habilitan y de cuáles no a los docentes. Algunos estilos directivos controlan más la comunicación con las familias, siempre con la finalidad de evitar consecuencias negativas que puedan surgir por la inmediatez de los mensajes digitales. Las reticencias de los directores a liberar estos canales tienen que ver con las debilidades en competencias comunicacionales de los docentes y las inseguridades de los directores por su falta de desarrollo en esas mismas competencias. Algunos maestros señalan el peso de la tradición o el miedo a lo novedoso como un motivo adicional de este comportamiento.

El cuaderno de comunicados y las entrevistas personales continúan siendo los canales preferidos de comunicación con las familias, aún en un contexto de utilización masiva de TIC. Una de las razones más mencionadas se refiere a la percepción sobre la seguridad de la recuperación de "lo dicho" que otorga el papel, algo también posible con las TIC.

Podemos decir que las resistencias a la incorporación de tecnologías digitales para la comunicación son el resultado de varios factores: la cultura conservadora de la institución escolar, la falta de competencias digitales de los directores y maestros, y la existencia en algunos contextos de barreras socioeconómicas en al acceso a tecnología asequible y confiable por parte de las familias. Es real que no todos poseen (ni los docentes, ni las familias) celulares con la memoria suficiente para sumar una aplicación más, y esto excluye a una parte.

Las debilidades en la formación docente también aparecieron como un factor recurrente en el estudio. La escasa formación en competencias digitales, en general focalizada en el uso de las TIC en el aula, obvia la capacitación para la comunicación digital con las familias.

Pero no solo se encuentran resistencias o experiencias negativas. También se recogieron prácticas de comunicación digital y percepciones positivas, principalmente entre los docentes más jóvenes. Todo indicaría que, bien gestionados, los canales de comunicación digital podrían ser un recurso con un gran potencial para 
estrechar los vínculos con las familias, estableciendo sobre todo comunicaciones bidireccionales, lo que favorecería su implicación en los centros.

\section{Conclusiones}

¿Cómo pueden ser utilizados más eficazmente los canales digitales? Consideramos importante que cada centro pueda desarrollar un plan de comunicación, con objetivos precisos, que permitan definir qué canales van a utilizar para comunicarse con cada público y en qué circunstancias. La formulación de un protocolo, como parte del plan, puede ser la ocasión para involucrar a todos los actores en una visión más amplia acerca de la importancia de la comunicación bidireccional con las familias. Es primordial cambiar las actitudes y capacitar a directivos y docentes en competencias comunicativas e invertir en el desarrollo de competencias digitales, no solo con finalidades pedagógicas, sino como habilidades imprescindibles para la construcción de alianzas con las familias. Así se favorecerá la participación de las familias en la vida del centro y, por lo tanto, en el desarrollo de los aprendizajes de los alumnos.

Contextos actuales como el de la pandemia de covid-19 obligan -a posteriori- a invertir tiempo y recursos para el desarrollo de competencias comunicacionales y digitales. Tal como sugiere el Banco Interamericano de Desarrollo (2020), los centros educativos con capacidades instaladas para el uso de canales de comunicación virtuales o mediados por computadoras se encuentran en la actualidad en una posición más ventajosa para asegurar la continuidad educativa frente a la crisis provocada por el covid-19. En contraste, los centros que no lograron despegarse del cuaderno de comunicados como canal predilecto de comunicación se vieron impedidos de usarlo debido al distanciamiento social, lo que los hizo atravesar importantes dificultades para mantener su vínculo con su stakeholder clave: las familias (Wang, Zhang, Zhao, Zhang, y Jiang, 2020; Huang, Liu, Tlili, Yang, y Wang, 2020).
Este trabajo explora una muestra intencional en una red de centros educativos con características particulares: urbana, capitalina y privada. Si bien se considera que gran parte de los hallazgos son generalizables a otro tipo de centros y contextos regionales, se debería ampliar el universo de estudio y continuar investigando, por ejemplo, qué sucede en otros centros educativos privados que desarrollan sus actividades en otros contextos socioeconómicos y, por supuesto, en centros públicos. Sería adecuado continuar esta línea de investigación realizando estudios que permitan cuantificar, por ejemplo, los centros que establecen comunicación digital con las familias, cuáles son los canales más utilizados y cuáles son las debilidades y fortalezas más frecuentes.

Asimismo, fueron estudiados únicamente los extremos de la educación primaria, y es un debe extender la investigación a otros niveles como preescolar y secundaria. De particular importancia es, además, abordar las dinámicas de comunicación con otros stakeholders de los centros como, por ejemplo, entre directivos y docentes, desde un enfoque de funcionamiento organizacional interno.

\section{Referencias}

Aragonés, J. P. (2001). La competencia comunicativa en el entorno tecnológico: desafío para la enseñanza. Comunicar, (17), 21-30.

Banco Interamericano de Desarrollo. (2019). Del papel a la nube: Cómo guiar la transformación digital de los Sistemas de Información y Gestión Educativa (SIGED). Recuperado de: https:// publications.iadb.org/es/del-papel-la-nube-como-guiar-latransformacion-digital-de-los-sistemas-de-informacion-ygestion

Banco Interamericano de Desarrollo. (2020). La educación en tiempos del coronavirus: Los sistemas educativos de América Latina y el Caribe ante COVID-19. Recuperado de https:// publications.iadb.org/es/la-educacion-en-tiempos-del-coronavirus-los-sistemas-educativos-de-america-latina-y-el-caribe-ante-covid-19 
Beauchamp, L., y Parsons, J. (2014). Liderazgo instructivo en Alberta: Hallazgos de la investigación en cinco escuelas altamente eficaces. REICE: Revista lberoamericana sobre Calidad, Eficacia y Cambio en Educación, 12(4), 89-98.

Bochaca, J. G. (2015). La comunicación familia-escuela en educación infantil y primaria. Revista de Sociología de la Educación-RASE, 8(1), 71-85.

Bordalba, M. M. (2016). La comunicación familia-escuela: el uso de las TIC en los centros de primaria. Revista electrónica interuniversitaria de formación del profesorado, 19(1), 73-83.

Bouhnik, D., Deshen, M., y Gan, R. (2014). WhatsApp goes to school: Mobile instant messaging between teachers and students. Journal of Information Technology Education: Research, 13(1), 217-231.

Bush, T. (2007). Educational leadership and management: Theory, policy and practice. South African journal of education, 27(3), 391-406.

Calvo, J. J. (Coord.). (2013). Atlas sociodemográfico y de la desigualdad del Uruguay. Fascículo 1: Las necesidades básicas insatisfechas a partir de los Censos 2011. Recuperado de http://www.ine.gub.uy/documents/10181/34017/ Atlas_fasciculo_1_NBI_versionrevisada.pdf/57ea17f93fd9-4306-b9ca-948abc7fab73

Cox, D., y McLeod, S. (2014). Social media strategies for school principals. NASSP Bulletin, 98(1), 5-25.

Engen, B. K. (2019). Comprendiendo los aspectos culturales y sociales de las competencias digitales docentes. Comunicar: Revista científica iberoamericana de comunicación y educación, (61), 9-19.

El Nokali, N. E., Bachman, H. J., y Votruba-Drzal, E. (2010). Parent involvement and children's academic and social development in elementary school. Child development, 81(3), 988-1005.

Epstein, J. L. (1985). Home and school connections in schools of the future: Implications of research on parent involvement. Peabody Journal of Education, 62(2), 18-41.

Epstein, J. L. (1995). School/family/community partnerships. Phi delta kappan, 76(9), 701
Epstein, J. L. (2013). Preocupándonos por los niños que compartimos. En Programas efectivos de

involucramiento familiar en las escuelas: estudios y prácticas (pp.15-50). Recuperado de http://www.upla.cl/noticias/ wp-content/uploads/2015/09/capitulos_-seleccionados_ joyce_epstein.pdf

Ersöz, S. (2019). Understanding WhatsApp Parent Group's Dynamics: Group Communication and Information Sharing. Manas Sosyal Aratırmalar Dergisi, 8(1), 1167-1183.

Forero, C. P. S. (2011). Estado del arte de la nueva comunicación estratégica en Iberoamérica y Colombia. Signo y Pensamiento, 37(59), 234-346.

Fraillon, J., Ainley, J., Schulz, W., Friedman, T., y Duckworth, D. (2019). Preparing for life in a digital world: the IEA International Computer and Information Literacy Study 2018 International Report. Recuperado de https://www.iea.nl/sites/default/files/2019-11/ICILS\%202019\%20Digital\%20final\%20 04112019.pdf

Goodall, J. S. (2016). Technology and school-home communication. International Journal of Pedagogies and Learning, 11(2), 118-131.

Gore, E. (2004). La educación en la empresa: Aprendiendo en contextos organizativos ( $3^{\mathrm{a}} \mathrm{ed}$ ). Buenos Aires, Argentina: Granica.

Guardia Romero, R. M. D. L., y Santana Armas, F. (2010). Alternativas de mejora de la participación educativa de las familias como instrumento para la calidad educativa. REICE: Revista Electrónica Iberoamericana Sobre Calidad, Eficacia y Cambio En Educación, 8(3), 6-30.

Hernández, I. M., Martínez, A. C., y Vicente, M. A. G. (2017). Grupos de WhatsApp en familias de Educación Infantil y Primaria. RELATEC: Revista Latinoamericana de Tecnología Educativa, 16(2), 239-255.

Hodas, S. (1996). Technology refusal and the organizational culture of schools. Computerization and controversy: Value conflicts and social choices, 2, 197-218.

Hohlfeld, T. N., Ritzhaupt, A. D., y Barron, A. E. (2010). Connecting schools, community, and family with ICT: Four-year trends related to school level and SES of public schools in Florida. Computers \&t Education, 55(1), 391-405. 
Huang, R. H., Liu, D. J., Tlili, A., Yang, J. F., y Wang, H. H. (2020). Handbook on Facilitating Flexible Learning During Educational Disruption: The Chinese Experience in Maintaining Undisrupted Learning in COVID-19 Outbreak. Beijing, China: Smart Learning Institute of Beijing Normal University.

LaRocque, M., Kleiman, I., y Darling, S. M. (2011). Parental involvement: The missing link in school achievement. Preventing School Failure, 55(3), 115-122.

Lingam, G., Lingam, N., y Raghuwaiya, K. (2014). Effectiveness of school strategic planning: The case of Fijian Schools. International Journal of Social, Behavioral, Educational, Economic, Business and Industrial Engineering, 8(7), 2110-2116.

Mayo, I. C., y García, A. M. (2012). La comunicación en los centros educativos: un estudio de caso. Tendencias pedagógicas, (19), 107-130.

Noblia, V. (2018). La interacción laboral en redes sociales móviles. El uso de los modos como estrategia de atenuación. Círculo de Lingüística Aplicada a la Comunicación, 73, 77-102.

Odhiambo, G., y Hii, A. (2012). Key stakeholders' perceptions of effective school leadership. Educational Management Administration \& Leadership, 40(2), 232-247.

Olmstead, C. (2013). Using technology to increase parent involvement in schools. TechTrends, 57(6), 28-37.

Peña-López, I. (2009). Measuring digital development for policy-making: Models, stages, characteristics and causes (Tesis de Doctorado). Internet Interdisciplinary Institute, Universitat Oberta de Catalunya, Barcelona, España.

Pieri, M. (2018). Le relazioni scuola / famiglia in Italy: una ricerca in un Istituto Comprensivo dell'Emilia-Romagna. Annali en línea della Didattica e della Formazione Docente, 10(15-16), 464-478.

Reimers, F. M., y Schleicher, A. (2020). A framework to guide an education response to the COVID-19 Pandemic of 2020. Recuperado de https://www.hm.ee/sites/default/files/framework_guide_v1_002_harward.pdf

Robinson, L., Cotten, S. R., Ono, H., Quan-Haase, A., Mesch, G., Chen, W., ... Stern, M. J. (2015). Digital inequalities and why they matter. Information, communication \& society, 18(5), 569-582.
Walther, J. B. (1996). Computer-mediated communication: Impersonal, interpersonal, and hyperpersonal interaction. Communication research, 23(1), 3-43.

Walther, J. B. (2011). Theories of computer-mediated communication and interpersonal relations. The handbook of interpersonal communication, 4, 443-479.

Wang, G., Zhang, Y., Zhao, J., Zhang, J., y Jiang, F. (2020). Mitigate the effects of home confinement on children during the COVID-19 outbreak. The Lancet, 395(10228), 945-947.

Wasserman, E., y Zwebner, Y. (2017). Communication between teachers and parents using the WhatsApp application. International Journal of Learning, Teaching and Educational Research, 16(12). https://doi.org/10.26803/ijlter.16.12.1

Wellman, B., Quan-Haase, A., Boase, J., Chen, W., Hampton, K., Diaz, I., y Miyata, K. (2003). The social affordances of the Internet for networked individualism. Journal of computer-mediated communication, 8(3), JCMC834.

Wellman, B., y Hogan, B. (2004). The immanent internet. En J. McKay (ed.), Netting citizens: Exploring citizenship in a digital age (pp. 54-80). Edimburgo, Escocia: St. Andrews Press.

\section{Conflicto de interés}

Debido a que uno o más miembros de los miembros del equipo de investigación se encuentra vinculado a uno de los centros de la red estudiado, para evitar conflictos se prestó particular atención a que las entrevistas siempre fueran realizadas por entrevistadores ajenos a los centros educativos.

\section{Contribución autoral}

a) Concepción y diseño del trabajo; b) Adquisición de datos; c) Análisis e interpretación de datos; d) Redacción del manuscrito; e) revisión crítica del manuscrito.

\section{P. S. ha contribuido en $a, b, c, d$, e.}

M. D. S. ha contribuido en a, e.

A. I. P. ha contribuido en a, e.

Editor responsable: Leandro Delgado 